\title{
The concavity and convexity of the Boros-Moll sequences
}

\author{
Ernest X.W. Xia* \\ Department of Mathematics \\ Jiangsu University \\ Zhenjiang, Jiangsu 212013, P.R. China \\ ernestxwxia@163.com
}

Submitted: Oct 21, 2013; Accepted: Dec 17, 2014; Published: Jan 9, 2015

Mathematics Subject Classifications: 05A20, 05A10, 11B83

\begin{abstract}
In their study of a quartic integral, Boros and Moll discovered a special class of sequences, which is called the Boros-Moll sequences. In this paper, we consider the concavity and convexity of the Boros-Moll sequences $\left\{d_{i}(m)\right\}_{i=0}^{m}$. We show that for any integer $m \geqslant 6$, there exist two positive integers $t_{0}(m)$ and $t_{1}(m)$ such that $d_{i}(m)+d_{i+2}(m)>2 d_{i+1}(m)$ for $i \in\left[0, t_{0}(m)\right] \bigcup\left[t_{1}(m), m-2\right]$ and $d_{i}(m)+d_{i+2}(m)<$ $2 d_{i+1}(m)$ for $i \in\left[t_{0}(m)+1, t_{1}(m)-1\right]$. When $m$ is a square, we find $t_{0}(m)=\frac{m-\sqrt{m}-4}{2}$ and $t_{1}(m)=\frac{m+\sqrt{m}-2}{2}$. As a corollary of our results, we show that

$$
\lim _{m \rightarrow+\infty} \frac{\operatorname{card}\left\{i \mid d_{i}(m)+d_{i+2}(m)<2 d_{i+1}(m), 0 \leqslant i \leqslant m-2\right\}}{\sqrt{m}}=1 .
$$
\end{abstract}

Keywords: Boros-Moll sequences; concavity; convexity; log-concavity; log-convexity

\section{Introduction and Main Results}

The object of this paper is to study the concavity and convexity of the Boros-Moll sequences. Boros and Moll [4, 5, 6, 7, 8] explored a special class of Jacobi polynomials in their study of a quartic integral. They have shown that for any $a>-1$ and any nonnegative integer $m$,

$$
\int_{0}^{\infty} \frac{1}{\left(x^{4}+2 a x^{2}+1\right)^{m+1}} d x=\frac{\pi}{2^{m+3 / 2}(a+1)^{m+1 / 2}} P_{m}(a)
$$

*This work was supported by the National Natural Science Foundation of China (11201188). 
where

$$
P_{m}(a)=\sum_{j, k}\left(\begin{array}{c}
2 m+1 \\
2 j
\end{array}\right)\left(\begin{array}{c}
m-j \\
k
\end{array}\right)\left(\begin{array}{c}
2 k+2 j \\
k+j
\end{array}\right) \frac{(a+1)^{j}(a-1)^{k}}{2^{3(k+j)}} .
$$

Using Ramanujan's Master Theorem, Boros and Moll [7, 18] derived the following formula

$$
P_{m}(a)=2^{-2 m} \sum_{k} 2^{k}\left(\begin{array}{c}
2 m-2 k \\
m-k
\end{array}\right)\left(\begin{array}{c}
m+k \\
k
\end{array}\right)(a+1)^{k}
$$

which indicates that the coefficients of $a^{i}$ in $P_{m}(a)$ are positive for $0 \leqslant i \leqslant m$. Chen, Pang and $\mathrm{Qu}[12]$ gave a combinatorial proof to show that $(2)$ is equal to $(3)$. Let $d_{i}(m)$ be defined by

$$
P_{m}(a)=\sum_{i=0}^{m} d_{i}(m) a^{i}
$$

The polynomials $P_{m}(a)$ will be called the Boros-Moll polynomials, and the sequences $\left\{d_{i}(m)\right\}_{i=0}^{m}$ of the coefficients will be called the Boros-Moll sequences. It follows from (3) and (4) that

$$
d_{i}(m)=2^{-2 m} \sum_{k=i}^{m} 2^{k}\left(\begin{array}{c}
2 m-2 k \\
m-k
\end{array}\right)\left(\begin{array}{c}
m+k \\
k
\end{array}\right)\left(\begin{array}{l}
k \\
i
\end{array}\right) .
$$

The readers can find in [3] many proofs of this formula. Recall that $P_{m}(a)$ can be expressed as a hypergeometric function

$$
P_{m}(a)=2^{-2 m}\left(\begin{array}{c}
2 m \\
m
\end{array}\right){ }_{2} F_{1}\left(-m, m+1 ; \frac{1}{2}-m ; \frac{a+1}{2}\right),
$$

from which one sees that $P_{m}(a)$ can be viewed as the Jacobi polynomial $P_{m}^{(\alpha, \beta)}(a)$ with $\alpha=m+\frac{1}{2}$ and $\beta=-\left(m+\frac{1}{2}\right)$, where $P_{m}^{(\alpha, \beta)}(a)$ is given by

$$
P_{m}^{(\alpha, \beta)}(a)=\sum_{k=0}^{m}(-1)^{m-k}\left(\begin{array}{c}
m+\beta \\
m-k
\end{array}\right)\left(\begin{array}{c}
m+k+\alpha+\beta \\
k
\end{array}\right)\left(\frac{1+a}{2}\right)^{k} .
$$

Some combinatorial properties of the Boros-Moll sequences have been established. Boros and Moll [5] proved that the sequence $\left\{d_{i}(m)\right\}_{i=0}^{m}$ is unimodal and the maximum element appears in the middle, namely,

$$
d_{0}(m)<d_{1}(m)<\cdots<d_{\left[\frac{m}{2}\right]}(m)>d_{\left[\frac{m}{2}\right]+1}(m)>\cdots>d_{m}(m) .
$$

They also established the unimodality of the sequence $\left\{d_{i}(m)\right\}_{i=0}^{m}$ by taking a different approach [6]. Amdeberhan, Dixit, Guan, Jiu and Moll [1] presented another proof of (8). Amdeberhan, Manna and Moll [2] analyzed properties of the 2-adic valuation of an 
integer sequence and gave a combinatorial interpretation of the valuations of the integer sequence which is related to the Boros-Moll sequences. Moll [18] conjectured that the sequence $\left\{d_{i}(m)\right\}_{i=0}^{m}$ is log-concave. Kauers and Paule [16] proved this conjecture based on the following four recurrence relations found by using the WZ-method [20]:

$$
\begin{aligned}
d_{i}(m+1)= & \frac{m+i}{m+1} d_{i-1}(m)+\frac{(4 m+2 i+3)}{2(m+1)} d_{i}(m), \quad 0 \leqslant i \leqslant m+1, \\
d_{i}(m+1)= & \frac{(4 m-2 i+3)(m+i+1)}{2(m+1)(m+1-i)} d_{i}(m) \\
& \quad-\frac{i(i+1)}{(m+1)(m+1-i)} d_{i+1}(m), \quad 0 \leqslant i \leqslant m, \\
d_{i}(m+2)= & \frac{-4 i^{2}+8 m^{2}+24 m+19}{2(m+2-i)(m+2)} d_{i}(m+1) \\
& \quad-\frac{(m+i+1)(4 m+3)(4 m+5)}{4(m+2-i)(m+1)(m+2)} d_{i}(m), \quad 0 \leqslant i \leqslant m+1,
\end{aligned}
$$

and for $0 \leqslant i \leqslant m+1$,

$$
\begin{aligned}
(m+2-i)(m+i-1) & d_{i-2}(m) \\
& -(i-1)(2 m+1) d_{i-1}(m)+i(i-1) d_{i}(m)=0 .
\end{aligned}
$$

In fact, the recurrences (11) and (12) are also derived independently by Moll [19] by using the WZ-method [20]. Chen and Gu [11] showed that the Boros-Moll sequences satisfy the reverse ultra log-concavity. Chen and Xia [13] proved that the Boros-Moll sequences satisfy the ratio monotone property which implies the log-concavity and the spiral property. They [14] also confirmed a conjecture given by Moll in [19]. By constructing an intermediate function, Chen and Xia [15] proved the 2-log-concavity of the Boros-Moll sequences. Chen, Dou and Yang [10] proved two conjectures of Brändén [9] on the real-rootedness of the polynomials $Q_{n}(x)$ and $R_{n}(x)$ which are related to the Boros-Moll polynomials $P_{n}(x)$. The first conjecture implies the 2-log-concavity of the Boros-Moll sequences, and the second conjecture implies the 3-log-concavity of the Boros-Moll sequences.

In this paper, we consider the concavity and convexity of the Boros-Moll sequences. Let $\left\{a_{i}\right\}_{i=0}^{n}$ be a sequence of real numbers. Recall that the sequence $\left\{a_{i}\right\}_{i=0}^{n}$ is said to be convex (resp. concave) if

$$
a_{i}+a_{i+2} \geqslant 2 a_{i+1} \quad\left(\text { resp. } \quad a_{i}+a_{i+2} \leqslant 2 a_{i+1}\right)
$$

for $0 \leqslant i \leqslant n-2$. It is easy to see that for positive sequences, the log-convexity implies the convexity and the concavity implies the log-concavity.

The main results of this paper can be stated as follows.

Theorem 1. Let $m, i$ be integers and $m \geqslant 6$. We have $d_{i}(m)+d_{i+2}(m)>2 d_{i+1}(m)$ for $i \in\left[0, t_{0}(m)\right] \bigcup\left[t_{1}(m), m-2\right]$ and $d_{i}(m)+d_{i+2}(m)<2 d_{i+1}(m)$ for $i \in\left[t_{0}(m)+1, t_{1}(m)-1\right]$, 
where $t_{0}(m)=\frac{m}{2}-\frac{\sqrt{m}}{2}-2$ and $t_{1}(m)=\frac{m}{2}+\frac{\sqrt{m}}{2}-1$ when $m$ is a square; $t_{0}(m)=\left[\frac{m}{2}-\frac{\sqrt{m}}{2}-2\right]$ or $\left[\frac{m}{2}-\frac{\sqrt{m}}{2}-1\right]$ and $t_{1}(m)=\left[\frac{m}{2}+\frac{\sqrt{m}}{2}-1\right]$ or $\left[\frac{m}{2}+\frac{\sqrt{m}}{2}\right]$ when $m$ is not a square.

In order to prove Theorem 1, we establish the following two Theorems:

Theorem 2. Let $m, i$ be integers and $m \geqslant 6$. We have $d_{i}(m)+d_{i+2}(m)>2 d_{i+1}(m)$ for $i \in\left[0, \frac{m}{2}-\frac{\sqrt{m}}{2}-2\right] \bigcup\left[\frac{m}{2}+\frac{\sqrt{m}}{2}-1, m-2\right]$.

Theorem 3. Let $m, i$ be integers and $m \geqslant 6$. We have $d_{i}(m)+d_{i+2}(m)<2 d_{i+1}(m)$ for $i \in\left[\frac{m}{2}-\frac{\sqrt{m}}{2}-1, \frac{m}{2}+\frac{\sqrt{m}}{2}-2\right]$.

Note that $\frac{m}{2}-\frac{\sqrt{m}}{2}$ is an integer if and only if $m$ is a square. Therefore, from Theorems 2 and 3, we immediately prove Theorem 1 . By Theorems 2 and 3, we can obtain the following corollary:

Corollary 4. We have

$$
\lim _{m \rightarrow+\infty} \frac{\operatorname{card}\left\{i \mid d_{i}(m)+d_{i+2}(m)<2 d_{i+1}(m), 0 \leqslant i \leqslant m-2\right\}}{\sqrt{m}}=1 .
$$

To conclude this section, we propose an open problem. Determine the signs of the differences $d_{\left[\frac{m}{2}-\frac{\sqrt{m}}{2}-1\right]}(m)+d_{\left[\frac{m}{2}-\frac{\sqrt{m}}{2}+1\right]}(m)-2 d_{\left[\frac{m}{2}-\frac{\sqrt{m}}{2}\right]}(m)$ and $d_{\left[\frac{m}{2}+\frac{\sqrt{m}}{2}-1\right]}(m)+d_{\left[\frac{m}{2}+\frac{\sqrt{m}}{2}+1\right]}(m)-$ $2 d_{\left[\frac{m}{2}+\frac{\sqrt{m}}{2}\right]}(m)$ when $m$ is not a square.

\section{Proofs of the Main Results}

In this section, we present proofs of the main results. We first represent $d_{i}(m)+d_{i+2}(m)-$ $2 d_{i+1}(m)$ in terms of $d_{i}(m)$ and $d_{i}(m+1)$.

Lemma 5. For $1 \leqslant i \leqslant m-2$, we have

$$
d_{i}(m)+d_{i+2}(m)-2 d_{i+1}(m)=A(m, i) d_{i}(m+1)+B(m, i) d_{i}(m)
$$

where

$$
\begin{aligned}
& A(m, i)=-\frac{(m+1)(m+1-i)(2 m-2 i-3)}{i(i+1)(i+2)} \\
& B(m, i)=\frac{\left(8 m^{3}-15 m-5 i-8 m i^{2}-6 m^{2} i-20 m i+2 m^{2}+12 i^{2}+8 i^{3}-9\right)}{2 i(i+1)(i+2)} .
\end{aligned}
$$

Proof. It follows from (10) and (12) that for $1 \leqslant i \leqslant m-1$,

$$
d_{i+1}(m)=\frac{(4 m-2 i+3)(m+i+1)}{2 i(i+1)} d_{i}(m)-\frac{(m+1-i)(m+1)}{i(i+1)} d_{i}(m+1)
$$


and

$$
d_{i+2}(m)=\frac{2 m+1}{i+2} d_{i+1}(m)-\frac{(m-i)(m+i+1)}{(i+1)(i+2)} d_{i}(m) .
$$

Lemma 5 follows from (18) and (19). This completes the proof.

Now, we are ready to prove Theorem 2.

Proof of Theorem 2. It is a routine to verify that Theorem 2 holds for $6 \leqslant m \leqslant 9$. So, we can assume that $m \geqslant 10$. It is easy to check that for $1 \leqslant i \leqslant m-2$,

$$
\begin{aligned}
-2(m+1)(m+1-i) \frac{B(m, i)}{A(m, i)} & -\left(4 m^{2}+7 m+3-2 i^{2}\right) \\
& =\frac{i\left(4 i^{2}-4 i m+2 m^{2}+6 i-6 m+1\right)}{(2 m-2 i-3)}>0 .
\end{aligned}
$$

It is easy to verify that

$$
\left(\frac{i\left(4 i^{2}-4 i m+2 m^{2}+6 i-6 m+1\right)}{(2 m-2 i-3)}\right)^{2}-i^{2}\left(4 m+4 i^{2}+1\right)=\frac{4 i^{2} G(m, i)}{(2 m-2 i-3)^{2}}
$$

where

$$
\begin{aligned}
G(m, i)= & \left(4 m^{2}-16 m+1\right) i^{2}-\left(4 m^{3}-26 m^{2}+30 m\right) i \\
& +m^{4}-10 m^{3}+21 m^{2}-9 m-2
\end{aligned}
$$

In Section 3, we will prove that for $i \in\left[0, \frac{m}{2}-\frac{\sqrt{m}}{2}-2\right] \bigcup\left[\frac{m}{2}+\frac{\sqrt{m}}{2}-1, m-2\right]$ and $m \geqslant 10$,

$$
G(m, i) \geqslant 0 \text {. }
$$

Combining (20), (21) and (23), we deduce that

$$
\frac{i\left(4 i^{2}-4 i m+2 m^{2}+6 i-6 m+1\right)}{(2 m-2 i-3)}>i \sqrt{4 m+4 i^{2}+1} .
$$

It follows from (20) and (24) that for $i \in\left[1, \frac{m}{2}-\frac{\sqrt{m}}{2}-2\right] \bigcup\left[\frac{m}{2}+\frac{\sqrt{m}}{2}-1, m-2\right]$ and $m \geqslant 10$,

$$
-\frac{B(m, i)}{A(m, i)}>\frac{4 m^{2}+7 m+3-2 i^{2}+i \sqrt{4 m+4 i^{2}+1}}{2(m+1)(m+1-i)} .
$$

In order to establish the reverse ultra log-concavity of $\left\{d_{i}(m)\right\}_{i=0}^{m}$, Chen and $\mathrm{Gu}$ [11] gave an upper bound of the ratio $d_{i}(m+1) / d_{i}(m)$. They proved that for $m \geqslant 2$ and $0 \leqslant i \leqslant m$,

$$
\frac{d_{i}(m+1)}{d_{i}(m)} \leqslant \frac{4 m^{2}+7 m+3-2 i^{2}+i \sqrt{4 m+4 i^{2}+1}}{2(m+1)(m+1-i)} .
$$


By (25) and (26), we see that for $i \in\left[1, \frac{m}{2}-\frac{\sqrt{m}}{2}-2\right] \bigcup\left[\frac{m}{2}+\frac{\sqrt{m}}{2}-1, m-2\right]$ and $m \geqslant 10$,

$$
-\frac{B(m, i)}{A(m, i)}>\frac{d_{i}(m+1)}{d_{i}(m)} \text {. }
$$

It should be noted that $A(m, i)<0$ for $1 \leqslant i \leqslant m-1$. Thus, the above inequality can be rewritten as

$$
A(m, i) d_{i}(m+1)+B(m, i) d_{i}(m)>0 .
$$

By Lemma 5 and (28), we see that $d_{i}(m)+d_{i+2}(m)-2 d_{i+1}(m)>0$ for $m \geqslant 10$ and $i \in\left[1, \frac{m}{2}-\frac{\sqrt{m}}{2}-2\right] \bigcup\left[\frac{m}{2}+\frac{\sqrt{m}}{2}-1, m-2\right]$.

It remains to verify that $d_{0}(m)+d_{2}(m)>2 d_{1}(m)$ for $m \geqslant 10$. In (12), let $i=2$, we have

$$
d_{0}(m)=\frac{2 m+1}{m(m+1)} d_{1}(m)-\frac{2}{m(m+1)} d_{2}(m) .
$$

Therefore,

$$
d_{0}(m)+d_{2}(m)-2 d_{1}(m)=\frac{m^{2}+m-2}{m(m+1)} d_{2}(m)-\frac{2 m^{2}-1}{m(m+1)} d_{1}(m) .
$$

Employing (5), we deduce that

$$
d_{m-2}(m)=\frac{(m-1)\left(4 m^{2}+2 m+1\right)}{2^{m+2}(2 m-1)}\left(\begin{array}{c}
2 m \\
m
\end{array}\right)
$$

and

$$
d_{m-3}(m)=2^{-m} \frac{(m-2)(2 m+1)\left(4 m^{2}-2 m+3\right)}{24(2 m-1)}\left(\begin{array}{c}
2 m \\
m
\end{array}\right) .
$$

By (31), (32) and the ratio monotone property of the Boros-Moll sequences established by Chen and Xia in [13], we have

$$
\frac{d_{2}(m)}{d_{1}(m)}>\frac{d_{m-3}(m)}{d_{m-2}(m)}=\frac{(m-2)(2 m+1)\left(4 m^{2}-2 m+3\right)}{6(m-1)\left(4 m^{2}+2 m+1\right)}>\frac{2 m^{2}-1}{m^{2}+m-2},
$$

which implies that the left hand side of $(30)$ is positive for $m \geqslant 10$. This completes the proof.

Now we turn to prove Theorem 3.

Proof of Theorem 3. It is easy to check that Theorem 3 is true for $6 \leqslant m \leqslant 931$ by Maple. In the following, we assume that $m \geqslant 932$. It is easy to verify that

$$
\begin{aligned}
& \frac{4 m^{2}+7 m+\frac{m}{i+2} i+4}{2(m+1)(m+1-i)}+\frac{B(m, i)}{A(m, i)} \\
= & \frac{-H(m, i)}{2(m+1)(m+1-i)(i+2)(2 m-2 i-3)},
\end{aligned}
$$


where

$$
\begin{aligned}
H(m, i)= & 8 i^{4}-8 i^{3} m+2 i^{2} m^{2}+28 i^{3}-20 i^{2} m \\
& +2 i m^{2}+27 i^{2}-11 i m+9 i-4 m+6 .
\end{aligned}
$$

We can prove that for $m \geqslant 932$ and $i \in\left[\frac{m}{2}-\frac{\sqrt{m}}{2}-1, \frac{m}{2}+\frac{\sqrt{m}}{2}-2\right]$,

$$
H(m, i)<0 .
$$

The proof of (36) is analogous to the proof of (23), and hence is omitted. In Section 4, we will prove that for $m \geqslant 55$ and $\left[\frac{2 m}{5}\right]+1 \leqslant i \leqslant m-1$,

$$
\frac{d_{i}(m+1)}{d_{i}(m)} \geqslant \frac{4 m^{2}+7 m+\frac{m}{i+2} i+4}{2(m+1)(m+1-i)} .
$$

In view of (34), (36) and (37), we find that for $i \in\left[\frac{m}{2}-\frac{\sqrt{m}}{2}-1, \frac{m}{2}+\frac{\sqrt{m}}{2}-2\right]$ and $m \geqslant 932$,

$$
\frac{d_{i}(m+1)}{d_{i}(m)} \geqslant \frac{4 m^{2}+7 m+\frac{m}{i+2} i+4}{2(m+1)(m+1-i)}>-\frac{B(m, i)}{A(m, i)},
$$

which implies

$$
A(m, i) d_{i}(m+1)+B(m, i) d_{i}(m)<0 .
$$

This is because $A(m, i)<0$ for $1 \leqslant i \leqslant m-1$. In view of Lemma 5 and (39), we deduce that $d_{i}(m)+d_{i+2}(m)-2 d_{i+1}(m)<0$ for $i \in\left[\frac{m}{2}-\frac{\sqrt{m}}{2}-1, \frac{m}{2}+\frac{\sqrt{m}}{2}-2\right]$ and $m \geqslant 932$. This completes the proof.

Proof of Corollary 4. It follows from Theorem 2 that

$$
\lim _{m \rightarrow+\infty} \frac{\operatorname{card}\left\{i \mid d_{i}(m)+d_{i+2}(m)<2 d_{i+1}(m), 0 \leqslant i \leqslant m-2\right\}}{\sqrt{m}} \leqslant 1 .
$$

Theorem 3 implies that

$$
\lim _{m \rightarrow+\infty} \frac{\operatorname{card}\left\{i \mid d_{i}(m)+d_{i+2}(m)<2 d_{i+1}(m), 0 \leqslant i \leqslant m-2\right\}}{\sqrt{m}} \geqslant 1 .
$$

Corollary 4 follows from (40) and (41). The proof is complete.

\section{Proof of (23)}

In this section, we present a proof of (23).

It is a routine to verify that for $m \geqslant 10$,

$$
\begin{aligned}
& 2\left(4 m^{2}-16 m+1\right)\left(\frac{m}{2}-\frac{\sqrt{m}}{2}-2\right)-\left(4 m^{3}-26 m^{2}+30 m\right) \\
= & -4 m^{5 / 2}-6 m^{2}+16 m^{3 / 2}+35 m-m^{1 / 2}-4<0 .
\end{aligned}
$$


Also, it is easy to check that for $m \geqslant 6$,

$$
\begin{aligned}
& 2\left(4 m^{2}-16 m+1\right)\left(\frac{m}{2}+\frac{\sqrt{m}}{2}-1\right)-\left(4 m^{3}-26 m^{2}+30 m\right) \\
= & 4 m^{5 / 2}+2 m^{2}-16 m^{3 / 2}+3 m+m^{1 / 2}-2>0 .
\end{aligned}
$$

It follows from (42) and (43) that for $m \geqslant 10$,

$$
\frac{m}{2}-\frac{\sqrt{m}}{2}-2<\frac{4 m^{3}-26 m^{2}+30 m}{2\left(4 m^{2}-16 m+1\right)}<\frac{m}{2}+\frac{\sqrt{m}}{2}-1 \text {. }
$$

It should be noted that $4 m^{2}-16 m+1>0$ for $m \geqslant 6$. Therefore, for $m \geqslant 10$ and $i \in\left[0, \frac{m}{2}-\frac{\sqrt{m}}{2}-2\right] \bigcup\left[\frac{m}{2}+\frac{\sqrt{m}}{2}-1, m-2\right]$, we obtain

$$
G(m, i) \geqslant \min \left\{G\left(m, \frac{m}{2}-\frac{\sqrt{m}}{2}-2\right), G\left(m, \frac{m}{2}+\frac{\sqrt{m}}{2}-1\right)\right\} .
$$

It is easy to verify that for $m \geqslant 10$,

$$
G\left(m, \frac{m}{2}-\frac{\sqrt{m}}{2}-2\right)=3 m^{5 / 2}-\frac{7}{4} m^{2}-\frac{35}{2} m^{3 / 2}-\frac{59}{4} m+2 m^{1 / 2}+2>0
$$

and

$$
G\left(m, \frac{m}{2}+\frac{\sqrt{m}}{2}-1\right)=m^{5 / 2}-\frac{15}{4} m^{2}+\frac{3}{2} m^{3 / 2}+\frac{17}{4} m-m^{1 / 2}-1>0 .
$$

Inequality (23) follows from (45), (46) and (47). This completes the proof.

\section{Proof of (37)}

In this section, we provide a proof of (37).

We are ready to prove (37) by induction on $m$. It is easy to check that (37) holds for $m=55$. We assume that (37) is true for $n \geqslant 55$, i.e.,

$$
\frac{d_{i}(n+1)}{d_{i}(n)} \geqslant \frac{4 n^{2}+7 n+\frac{n}{i+2} i+4}{2(n+1-i)(n+1)}, \quad\left[\frac{2 n}{5}\right]+1 \leqslant i \leqslant n-1 .
$$

We aim to prove that (37) holds for $n+1$, that is,

$$
\frac{d_{i}(n+2)}{d_{i}(n+1)} \geqslant \frac{4(n+1)^{2}+7(n+1)+\frac{n+1}{i+2} i+4}{2(n+2-i)(n+2)}, \quad\left[\frac{2(n+1)}{5}\right]+1 \leqslant i \leqslant n .
$$

It is easy to check that

$$
\begin{aligned}
& \frac{4 n^{2}+7 n+\frac{n}{i+2} i+4}{2(n+1-i)(n+1)}-\frac{(2+i)(4 n+5)(4 n+3)(n+i+1)}{-2\left(8 i^{2}+4 i^{3}-8 n^{2}-4 n^{2} i-18 n-8 n i-8-3 i\right)(n+1)} \\
= & \frac{F(n, i)}{2\left(8 n^{2}+4 n^{2} i+18 n+8 n i+8+3 i-8 i^{2}-4 i^{3}\right)(2+i)(n+1)(n+1-i)},
\end{aligned}
$$


where $F(n, i)$ is given by

$$
F(n, i)=4-4 i+8 n-7 i^{2}-4 n^{2} i-3 n^{2} i^{2}-6 n i-4 i^{3}-i^{4}+10 n i^{2}+8 n i^{3} .
$$

Let $f(i)$ and $g(i)$ be defined by

$$
f(i)=8 n i-3 n^{2}-i^{2}, \quad g(i)=10 n i-4 n^{2}-4 i^{2} .
$$

For $\left[\frac{2 n+2}{5}\right]+1 \leqslant i \leqslant n-1$, we find that

$$
f(i) \geqslant f\left(\frac{2 n}{5}\right)=\frac{n^{2}}{25}, \quad g(i) \geqslant g\left(\frac{2 n}{5}\right)=-\frac{16 n^{2}}{25}
$$

In view of (51), (52) and (53), we deduce that for $\left[\frac{2 n+2}{5}\right]+1 \leqslant i \leqslant n-1$ and $n \geqslant 55$,

$$
\begin{aligned}
F(n, i) & =f(i) i^{2}+g(i) i+(8 n+4-4 i)-\left(7 i^{2}+6 n i\right) \\
& >\frac{1}{25} n^{2} i^{2}-\frac{16}{25} n^{2} i-13 n i \geqslant \frac{n i}{25}\left(\frac{2 n^{2}}{5}-16 n-325\right)>0 .
\end{aligned}
$$

It follows from (50) and (54) that for $\left[\frac{2 n+2}{5}\right]+1 \leqslant i \leqslant n-1$ and $n \geqslant 55$,

$$
\frac{4 n^{2}+7 n+\frac{n}{i+2} i+4}{2(n+1-i)(n+1)} \geqslant \frac{(2+i)(4 n+5)(4 n+3)(n+i+1)}{-2\left(8 i^{2}+4 i^{3}-8 n^{2}-4 n^{2} i-18 n-8 n i-8-3 i\right)(n+1)} .
$$

By (48) and (55), we deduce that for $\left[\frac{2 n+2}{5}\right]+1 \leqslant i \leqslant n-1$,

$$
\frac{d_{i}(n+1)}{d_{i}(n)} \geqslant \frac{(2+i)(4 n+5)(4 n+3)(n+i+1)}{-2\left(8 i^{2}+4 i^{3}-8 n^{2}-4 n^{2} i-18 n-8 n i-8-3 i\right)(n+1)} .
$$

It is a routine to verify that

$$
\begin{gathered}
\frac{(n+i+1)(4 n+3)(4 n+5)}{4(n+2-i)(n+1)(n+2)} \\
\frac{-4 i^{2}+8 n^{2}+24 n+19}{2(n+2-i)(n+2)}-\frac{4(n+1)^{2}+7(n+1)+\frac{n+1}{i+2} i+4}{2(n+2)(n+2-i)} \\
=\frac{(2+i)(4 n+5)(4 n+3)(n+i+1)}{2\left(8 n^{2}+4 n^{2} i+18 n+8 n i+8+3 i-8 i^{2}-4 i^{3}\right)(n+1)},
\end{gathered}
$$

which implies that

$$
\frac{-4 i^{2}+8 n^{2}+24 n+19}{2(n+2-i)(n+2)}-\frac{4(n+1)^{2}+7(n+1)+\frac{n+1}{i+2} i+4}{2(n+2)(n+2-i)}>0 .
$$


By (57), we can rewrite (56) as follows

$$
d_{i}(n+1) \geqslant \frac{\frac{(n+i+1)(4 n+3)(4 n+5)}{4(n+2-i)(n+1)(n+2)}}{\frac{-4 i^{2}+8 n^{2}+24 n+19}{2(n+2-i)(n+2)}-\frac{4(n+1)^{2}+7(n+1)+\frac{n+1}{i+2} i+4}{2(n+2)(n+2-i)}} d_{i}(n) .
$$

It follows from $(58)$ and $(59)$ that for $\left[\frac{2 n+2}{5}\right]+1 \leqslant i \leqslant n-1$,

$$
\begin{gathered}
\frac{-4 i^{2}+8 n^{2}+24 n+19}{2(n+2-i)(n+2)} d_{i}(n+1)-\frac{(n+i+1)(4 n+3)(4 n+5)}{4(n+2-i)(n+1)(n+2)} d_{i}(n) \\
\geqslant \frac{4(n+1)^{2}+7(n+1)+\frac{n+1}{i+2} i+4}{2(n+2-i)(n+2)} d_{i}(n+1) .
\end{gathered}
$$

By (11), we find that the left hand side of $(60)$ equals $d_{i}(n+2)$. Thus we have verified the inequality (49) for $\left[\frac{2 n+2}{5}\right]+1 \leqslant i \leqslant n-1$. It is still necessary to show that (49) is true for $i=n$, that is,

$$
\frac{d_{n}(n+2)}{d_{n}(n+1)} \geqslant \frac{4(n+1)^{2}+7(n+1)+\frac{n+1}{n+2} n+4}{4(n+2)} .
$$

By (5), we get

$$
d_{n}(n+1)=2^{-n-2}(2 n+3)\left(\begin{array}{c}
2 n+2 \\
n+1
\end{array}\right) .
$$

It follows from (31) and (62) that

$$
\frac{d_{n}(n+2)}{d_{n}(n+1)}=\frac{(n+1)\left(4 n^{2}+18 n+21\right)}{2(n+2)(2 n+3)} \geqslant \frac{4(n+1)^{2}+7(n+1)+\frac{n+1}{n+2} n+4}{4(n+2)},
$$

which yields (61). Hence the proof is complete by induction.

\section{Acknowledgements}

The author would like to thank the anonymous referee for valuable suggestions and comments.

\section{References}

[1] T. Amdeberhan, A. Dixit, X. Guan, L. Jiu, and V.H. Moll. The unimodality of a polynomial coming from a rational integral. Back to the original proof. J. Math. Anal. Appl., 420:1154-1166, 2014.

[2] T. Amdeberhan, D. Manna, and V.H. Moll. The 2-adic valuation of a sequence arising from a rational integral. J. Combin. Theory Ser. A, 115:1474-1486, 2008. 
[3] T. Amdeberhan and V.H. Moll. A formula for a quartic integral: a survey of old proofs and some new ones. Ramanujan J., 18:91-102, 2009.

[4] G. Boros and V.H. Moll. An integral hidden in Gradshteyn and Ryzhik. J. Comput. Appl. Math., 106:361-368, 1999.

[5] G. Boros and V.H. Moll. A sequence of unimodal polynomials. J. Math. Anal. Appl. 237:272-285, 1999.

[6] G. Boros and V.H. Moll. A criterion for unimodality. Electron. J. Combin. 6: R3, 1999.

[7] G. Boros and V.H. Moll. The double square root, Jacobi polynomials and Ramanujan's Master Theorem. J. Comput. Appl. Math., 130:337-344, 2001.

[8] G. Boros and V.H. Moll. Irresistible Integrals. Cambridge University Press, Cambridge, 2004.

[9] P. Brändén. Iterated sequences and the geometry of zeros. J. Reine Angew. Math. 658:115-131, 2011

[10] W.Y.C. Chen, D.Q.J. Dou, and A.L.B. Yang. Brändén's conjectures on the BorosMoll polynomials. Inter. Math. Res. Notices, 2013:4819-4828, 2013.

[11] W.Y.C. Chen and C.C.Y. Gu. The reverse ultra log-concavity of the Boros-Moll polynomials. Proc. Amer. Math. Soc., 137:3991-3998, 2009.

[12] W.Y.C. Chen, S.X.M. Pang, and E.X.Y. Qu. On the combinatorics of the Boros-Moll polynomials. Ramanujan J., 21:41-51, 2010.

[13] W.Y.C. Chen and E.X.W. Xia. The ratio monotonicity of Boros-Moll polynomials. Math. Comput., 78:2269-2282, 2009.

[14] W.Y.C. Chen and E.X.W. Xia. Proof of Moll's minimum conjecture. European J. Combin., 34:787-791, 2013.

[15] W.Y.C. Chen and E.X.W. Xia. 2-log-concavity of the Boros-Moll polynomials. Proc. Edinburgh Math. Soc., 56:701-722, 2013.

[16] M. Kauers and P. Paule. A computer proof of Moll's log-concavity conjecture. Proc. Amer. Math. Soc., 135:3847-3856, 2007.

[17] D.V. Manna and V.H. Moll. A remarkable sequence of integers. Expo. Math., 27:289-312, 2009.

[18] V.H. Moll. The evaluation of integrals: A personal story. Notices Amer. Math. Soc., 49:311-317, 2002.

[19] V.H. Moll. Combinatorial sequences arising from a rational integral. Online J. Anal. Combin., 2:Article 4, 2007.

[20] H.S. Wilf and D. Zeilberger. An algorithmic proof theory for hypergeometric (ordinary and " $q$ ") multisum/integral identities. Invent. Math., 108:575-633, 1992. 